\title{
Bradycardie sévère et asystolie lors d'induction au sévoflurane et sufentanil à faible dose : à propos de trois cas
}

\author{
[Low dose sufentanil and severe bradycardia during induction with sevoflurane: \\ a report of three cases]
}

Valérie Cardinal MD, René Martin MD FRCPC, Jean-Pierre Tétrault MD MSc FRCP, Marie-José Colas MD FRCPC, Linda Gagnon MD FRCPC, Yves Claprood MD FRCPC

\begin{abstract}
Objectif : Dans une étude clinique randomisée et à double insu utilisant du fentanyl ou du sufentanil à faible dose lors d'une induction au sévoflurane, nous avons observé trois cas de bradycardie sévère qui nous ont forcés à abandonner l'étude. Nous désirons présenter ces trois cas.

Éléments cliniques : L'induction de l'anesthésie a été réalisée chez les trois patientes selon une technique de respiration unique d'un mélange de sévoflurane $8 \%$ avec des débits de protoxyde 3 $\mathrm{L} \cdot \mathrm{min}^{-1}$ et d'oxygène de $3 \mathrm{~L} \cdot \mathrm{min}^{-1}$. Après la perte de conscience, une dose de sufentanil $\left(0,025 \mu \mathrm{g} \cdot \mathrm{kg}^{-1}\right.$ pour la première patiente et $0,1 \mu \mathrm{g} \cdot \mathrm{kg}^{-1}$ pour les deux autres patientes) a été administrée. La fréquence cardiaque a chuté à 38,40 et 42 battements $\cdot \mathrm{min}^{-1}$ respectivement, deux minutes après l'injection du sufentanil chez ces trois patientes, évoluant jusqu'à une asystolie chez la troisième. Toutes les patientes ont répondu à de l'atropine 0,6 mg iv.
\end{abstract}

Conclusion : L'association de sufentanil, même à faible dose, lors d'une induction au sévoflurane à respiration unique, peut être dangereuse en raison d'une possibilité de bradycardie et même d'asystolie.

Objectives: In a randomized double blind clinical study using low doses of fentanyl or sufentanil during sevoflurane induction, we observed three cases of severe bradycardia, forcing us to discontinue the study. We wish to describe these three cases.

Clinical data: Anesthesia was induced in the three patients, using a single breath technique consisting of a mixture of sevoflurane $8 \%$ with a nitrous oxide flow of $3 \mathrm{~L} \cdot \mathrm{min}^{-1}$ and an oxygen flow of $3 \mathrm{~L} \cdot \mathrm{min}^{-1}$. After the patients had lost consciousness, low dose sufentanil was administered $\left(0.025 \mu \mathrm{g} \cdot \mathrm{kg}^{-1}\right.$ for the first patient and $0.1 \mu \mathrm{g} \cdot \mathrm{kg}^{-1}$ for the other two patients). Heart rate fell to 38,40 and 42 beats $\cdot \mathrm{min}^{-1}$ respectively two minutes after the sufentanil injection in these three patients, and progressed to asystole in the third. All the patients responded to atropine $0.6 \mathrm{mg}$ iv.

Conclusion: The addition of sufentanil, even at very low doses, during anesthesia induction with sevoflurane using a single breath technique, may be dangerous because of possible bradycardia and even asystole.

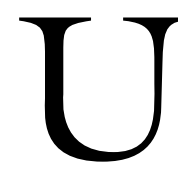

NE étude clinique avait été débutée afin de déterminer la dose de fentanyl et de sufentanil assurant la meilleure stabilité hémodynamique tout en offrant d'excellentes conditions d'intubation sans curare lors d'une induction à capacité vitale au sévoflurane. La survenue de deux bradycardies sévères et d'une asystolie en cours d'étude nous a forcés à y mettre fin prématurément. Nous présentons le cas des trois patientes ayant démontré des bradycardies importantes dont l'une a évolué à l'asystolie.

\section{Éléments cliniques}

Les patientes recrutées dans l'étude étaient soumises à une chirurgie programmée, étaient à jeun et n'avaient pas reçu de prémédication. Les patientes avaient été réparties en six groupes et recevaient, selon une technique de double insu, l'une des médications suivantes

Du Département d'anesthésiologie, Université de Sherbrooke, Sherbrooke, Québec, Canada.

Adresser la correspondance à : Dr Valérie Cardinal, Département d'anesthésiologie, CHUS, 3001 12e avenue nord, Sherbrooke,

Québec J1H 5N4, Canada. Téléphone : 819-346-1110, poste 14403; Télécopieur : 819-820-6413; Courriel : lise.gilbert@usherbrooke.ca

Présenté en partie au congrès annuel de la Société canadienne d'anesthésie, juin 2003.

Évalué le 11 novembre 2003.

Accepté le 2 mars 2004.

Révision acceptée le 26 avril 2004. 
soit : placebo, fentanyl $0,25 \mu \mathrm{g} \cdot \mathrm{kg}^{-1}$ ou $0,5 \mu \mathrm{g} \cdot \mathrm{kg}^{-1}$, sufentanil $0,025 \mu \mathrm{g} \cdot \mathrm{kg}^{-1}, 0,05 \mu \mathrm{g} \cdot \mathrm{kg}^{-1}$ ou $0,1 \mu \mathrm{g} \cdot \mathrm{kg}^{-1}$.

Un circuit en cercle semi-fermé saturé de sévoflurane à $8 \%$ avec des débits de $3 \mathrm{~L} \cdot \mathrm{min}^{-1} \mathrm{~d}^{\prime} \mathrm{O}_{2}$ et 3 $\mathrm{L} \cdot \mathrm{min}^{-1}$ de $\mathrm{N}_{2} \mathrm{O}$ était utilisé. Après deux minutes de préoxygénation, les patientes expiraient à volume résiduel puis inspiraient à capacité vitale le contenu du circuit. Après cinq respirations, la concentration de sévoflurane était diminuée à $6 \%$. Une voie veineuse était installée après la perte de conscience (avantage de cette méthode d'induction). La médication a été injectée dès que la voie veineuse était fonctionnelle. Quatre minutes plus tard, on intubait la trachée par laryngoscopie directe. La concentration de sévoflurane était alors diminuée à $2 \%$. La concentration expirée de sévoflurane n'a pas été enregistrée pendant l'étude. Trois des patientes recrutées ont présenté des bradycardies dont l'une, une asystolie.

\section{Cas \#1}

La première patiente était âgée de $5 \mathrm{l}$ ans et requérait une chirurgie gynécologique pour correction d'incontinence urinaire d'effort. Elle était connue pour une polyarthrite rhumatoïde contrôlée avec du naproxen au besoin. Elle ne fumait plus depuis 20 ans. Elle prenait une hormonothérapie de remplacement, du trazodone et du citalopram. Elle avait une tension artérielle (TA) de base de $154 / 81 \mathrm{mmHg}$ et un pouls de 59 battements. $\mathrm{min}^{-1}$. Le protocole d'induction au sévoflurane a été respecté. La patiente a reçu 0,025 $\mu \mathrm{g} \cdot \mathrm{kg}^{-1}(1,6 \mu \mathrm{g})$ de sufentanil quatre minutes après la première respiration de sévoflurane. Deux minutes après l'injection, la fréquence cardiaque $(\mathrm{FC})$ a chuté à 47 battements. $\mathrm{min}^{-1}$, de même que la TA à $73 / 32$ $\mathrm{mmHg}$. Cinq milligrammes d'éphédrine ont été injectés tel qu'il était prévu dans le protocole de recherche. Malgré cela, les signes vitaux se détériorent à la minute suivante : FC 40 et TA impossible à prendre au brassard. La patiente reçoit alors $0,6 \mathrm{mg}$ d'atropine, accélérant la FC à 90 et augmentant la TA à 127/75 mmHg. Le reste de l'anesthésie s'est déroulé sans particularité. La patiente a été évaluée en médecine interne la journée suivante pour une douleur thoracique atypique, avec apparition à l'électrocardiogramme d'une onde Q isolée en D3, laquelle a été jugée non significative, car les marqueurs biochimiques sont demeurés négatifs.

\section{Cas \#2}

Il s'agissait d'une dame de 42 ans se présentant pour une hystérectomie abdominale. Elle était considérée ASA II en raison d'une hypothyroïdie et d'un diabète de type II sous diète. Elle fumait dix cigarettes par jour et n'avait aucune allergie. Elle prenait de la lévothyroxine et de la venlafaxine. Elle présentait à l'entrée en salle une FC de 56 battements. $\mathrm{min}^{-1}$ et une TA de 123/64 mmHg. L'induction a été faite telle que décrite précédemment. La voie veineuse a été installée et le sufentanil $0,1 \mu \mathrm{g} \cdot \mathrm{kg}^{-1}(7,5 \mu \mathrm{g})$ a été injecté deux minutes après le début de l'induction. Deux minutes plus tard, la FC a diminué à 38 battements. $\mathrm{min}^{-1}$ et la $\mathrm{TA}$, à $61 / 26 \mathrm{mmHg}$. La patiente a bien répondu à une dose de $0,6 \mathrm{mg}$ d'atropine iv avec une augmentation de la FC à 94 battements. $\mathrm{min}^{-1}$. Le reste de l'anesthésie s'est déroulé normalement et la patiente a reçu son congé de l'hôpital quatre jours après la chirurgie.

\section{Cas \#3}

La troisième patiente, dernière de notre protocole, était âgée de 49 ans et se présentait pour une vulvectomie partielle. Au questionnaire, elle rapportait une hypotension lors des anesthésies précédentes, sans que celle-ci ne soit objectivée à la révision du dossier. Elle présentait un tabagisme actif et prenait du citalopram et des oestrogènes. Son électrocardiogramme avant la chirurgie était normal. Les signes vitaux avant l'induction montraient une FC à 64 battements $\mathrm{min}^{-1}$ et une TA de 124/52 mmHg. Il s'est écoulé cinq minutes entre la respiration initiale de sévoflurane et l'injection $i v$ de sufentanil $0,1 \mu \mathrm{g} \cdot \mathrm{kg}^{-1}(5,2 \mu \mathrm{g})$. Deux minutes après l'injection, la FC passe de 79 à 42 alors que la TA est à $90 / 50 \mathrm{mmHg}$. Une minute plus tard, soit trois minutes après avoir reçu le sufentanil, la patiente présente une asystolie. Un massage cardiaque (environ six compressions) a été initié immédiatement et de l'atropine $0,6 \mathrm{mg} i v$, injectée. Un rythme sinusal normal est réapparu au bout de $25 \mathrm{sec}$ et la chirurgie a pu être faite sans autre problème. La patiente a été extubée à la fin de la chirurgie et ne présentait aucune séquelle neurologique. Elle n'a pas été évaluée en cardiologie. Elle a subi par la suite une autre anesthésie générale au propofol et rémifentanil sans événement hémodynamique notable.

\section{Discussion}

Nous présentons le cas des trois patientes ayant présenté une bradycardie ou une asystolie au cours de l'étude proposée. À notre connaissance, aucune étude ni rapport de cas ne fait mention de bradycardie sévère avec l'association sévoflurane - sufentanil en technique d'induction à respiration unique à capacité vitale.

Le sévoflurane lui même n'a pas été démontré comme affectant la FC par rapport à l'état d'éveil, mais il peut causer de l'hypotension à plus haute dose $(1,5$ à $2,0 \mathrm{MAC})$, surtout en absence de stimulation 
chirurgicale. ${ }^{1}$ Par contre, Green et coll. ont démontré que, lors d'une induction par inhalation au sévoflurane chez des enfants, $20 \%$ de ceux-ci développaient un rythme nodal, lequel était observé surtout s'il y avait une bradycardie préexistante $(<80$ battements. $\left.\min ^{-1}\right) .{ }^{2}$ Townsend et Stokes, dans une lettre à l'éditeur, ${ }^{3}$ ont également rapporté quatre cas de bradycardie sévère chez des enfants de six mois à deux ans lors d'induction au sévoflurane $8 \%$ et $\mathrm{N}_{2} \mathrm{O} 66 \%$. Il est donc possible que le sévoflurane à lui seul puisse causer une bradycardie.

Le sufentanil est reconnu pour sa propriété à assurer une stabilité hémodynamique même à des doses élevées $\left(10 \mu \mathrm{g} \cdot \mathrm{kg}^{-1}{ }^{4}\right.$ et $\left.30 \mu \mathrm{g} \cdot \mathrm{kg}^{-1}\right) .{ }^{5}$ Néanmoins, des cas d'hypotension ont été rapportés à des doses plus faibles. ${ }^{6}$ De plus, l'augmentation du tonus vagal est un effet secondaire bien connu du sufentanil. Ainsi, une tendance à la bradycardie a été observée lors de l'association sufentanil à haute dose et vécuronium ${ }^{7}$ et des cas de bradycardie sévère et d'asystolie ont été rapportés. ${ }^{5-7}$ Le pancuronium semble protéger de la bradycardie associée au sufentanil par son effet sympatholytique, ${ }^{7,8}$ contrairement au glycopyrrolate donné en prémédication. ${ }^{6}$ Une étude fait également mention d'une incidence de $58 \%$ de bradycardie (diminution de plus de $20 \%$ de la FC) lors de l'association sévoflurane - sufentanil en chirurgie cardiaque, ${ }^{9}$ à des doses de $0,5 \mu \mathrm{g} \cdot \mathrm{kg}^{-1}$ en bolus suivie de $0,5 \mu \mathrm{g} \cdot \mathrm{kg}^{-1} \cdot \mathrm{min}^{-1}$ en perfusion. Cependant, $60 \%$ des patients de cette étude prenaient des ß-bloqueurs et $67 \%$ des bloqueurs calciques. Les épisodes de bradycardie semblent avoir été sans conséquence clinique.

D'autres cas de bradycardie ont été rapportés lors de l'association de sévoflurane et d'opioïdes. Ainsi, Wang et coll. ${ }^{10}$ ont fait mention d'un cas d'asystolie réanimé avec succès lors d'une induction au sévoflurane supplémentée, après la perte de conscience, de rémifentanil $0,5 \mu \mathrm{g} \cdot \mathrm{kg}^{-1}$ en bolus suivi d'une infusion de $0,025 \mu \mathrm{g} \cdot \mathrm{kg}^{-1} \cdot \mathrm{min}^{-1}$. Le patient avait une FC de base à 51 par minute secondairement à la prise d'aténolol et de diltiazem. De même, Kurdi et coll. ${ }^{11}$ ont rapporté un cas similaire chez un patient ne prenant aucune médication bradycardisante mais présentant une FC de 62 battements. $\mathrm{min}^{-1}$ à l'induction. L'hypothèse de base expliquant ces deux cas d'asystolie était un tonus vagal augmenté par le rémifentanil chez des patients ayant une réponse sympathique diminuée par le sévoflurane. Par contre, en considérant un facteur de puissance de 2 entre le sufentanil et le rémifentanil, la dose de rémifentanil utilisée en bolus $\left(0,5 \mu \mathrm{g} \cdot \mathrm{kg}^{-1}\right)$ était environ deux à trois fois plus élevée que la plus forte des doses de sufentanil que nous avons utilisées $\left(0,1 \mu \mathrm{g} \cdot \mathrm{kg}^{-1}\right)$.
Deux des trois patientes ayant présenté des bradycardies prenaient un inhibiteur sélectif du recaptage de la sérotonine, le citalopram. Ce médicament abaisse, en moyenne, la FC d'environ 5 battements. $\min ^{-1} .{ }^{12} \mathrm{Il}$ est par contre peu probable que cette diminution ait pu contribuer aux épisodes de bradycardie sévère que nous avons observés.

Finalement, notre principale hypothèse expliquant les bradycardies sévères rencontrées repose sur l'augmentation du tonus vagal par un effet synergique entre le sévoflurane et le sufentanil, lequel ne serait pas ou moins présent avec le fentanyl. Comme la FC et la TA au départ étaient légèrement plus basses chez les patientes ayant présenté des bradycardies, il est possible qu'un tonus vagal de base plus élevé ait pu ajouter à cette potentialisation. ${ }^{6}$

\section{Conclusion}

Une patiente, ayant reçu $0,1 \mu \mathrm{g} \cdot \mathrm{kg}^{-1}$ de sufentanil après une induction au masque avec la technique d'inspiration unique de sévoflurane, a présenté une asystolie. Deux autres patientes ayant reçu respectivement 0,025 et $0,1 \mu \mathrm{g} \cdot \mathrm{kg}^{-1}$ de sufentanil ont nécessité de l'atropine en réponse à une bradycardie < 40 . L'association de sufentanil lors d'une induction au sévoflurane peut être dangereuse en raison de la possibilité de bradycardie sévère et même d'asystolie.

\section{Références}

1 Malan TP Jr, DiNardo JA, Isner RJ, et al. Cardiovascular effects of sevoflurane compared with those of isoflurane in volunteers. Anesthesiology 1995; 83: 918-28.

2 Green DH, Townsend P, Bagshaw O, Stokes MA. Nodal rhythm and bradycardia during inhalation induction with sevoflurane in infants: a comparison of incremental and high-concentration techniques. Br J Anaesth 2000; 85: 368-70.

3 Townsend P, Stokes MA. Bradycardia during rapid inhalation induction with sevoflurane in children (Letter). Br J Anaesth 1998; 80: 410.

4 Sebel PS, Bovil JG. Cardiovascular effects of sufentanil anesthesia. Anesth Analg 1982; 61: 115-9.

5 Coda BA. Opioids. In: Barash PG, Cullen BF, Stoelting RK (Eds). Clinical Anesthesia, 4th ed. Philadelphia: Lippincott Williams \& Wilkins; 2001: 360.

6 Spiess BD, Sathoff RH, El-Ganzouri AR, Ivankovich $A D$. High-dose sufentanil: four cases of sudden hypotension on induction. Anesth Analg 1986; 65: 703-5.

7 Thomson IR, MacAdams CL, Hudson RJ, Rosenbloom $M$. Drug interactions with sufentanil. Hemodynamic 
effects of premedication and muscle relaxants. Anesthesiology 1992; 76: 922-9.

8 Starr NJ, Sethna DH, Estefanous FG. Bradycardia and asystole following the rapid administration of sufentanil with vecuronium. Anesthesiology 1986; 64: 521-3.

9 Gravel NR, Searle NR, Taillefer J, Carrier M, Roy M, Gagnon L. Comparison of the hemodynamic effects of sevoflurane anesthesia induction and maintenance vs TIVA in CABG surgery. Can J Anesth 1999; 46: 240-6.

10 Wang J, Winship S, Russel G. Induction of anaesthesia with sevoflurane and low-dose remifentanil: asystole following laryngoscopy (Letter). Br J Anaesth 1998; 81: 994-5.

11 Kurdi O, Deleuze A, Marret E, Bonnet F. Asystole during anaesthetic induction with remifentanil and sevoflurane (Letter). Br J Anaesth 2001; 87: 943.
12 Association des Pharmaciens du Canada. Compendium des Produits et Spécialités Pharmaceutiques. L'ouvrage de référence canadien sur les médicaments pour les professionnels de la santé. Ottawa, ON: Webcom Limitée; 2003: 371.

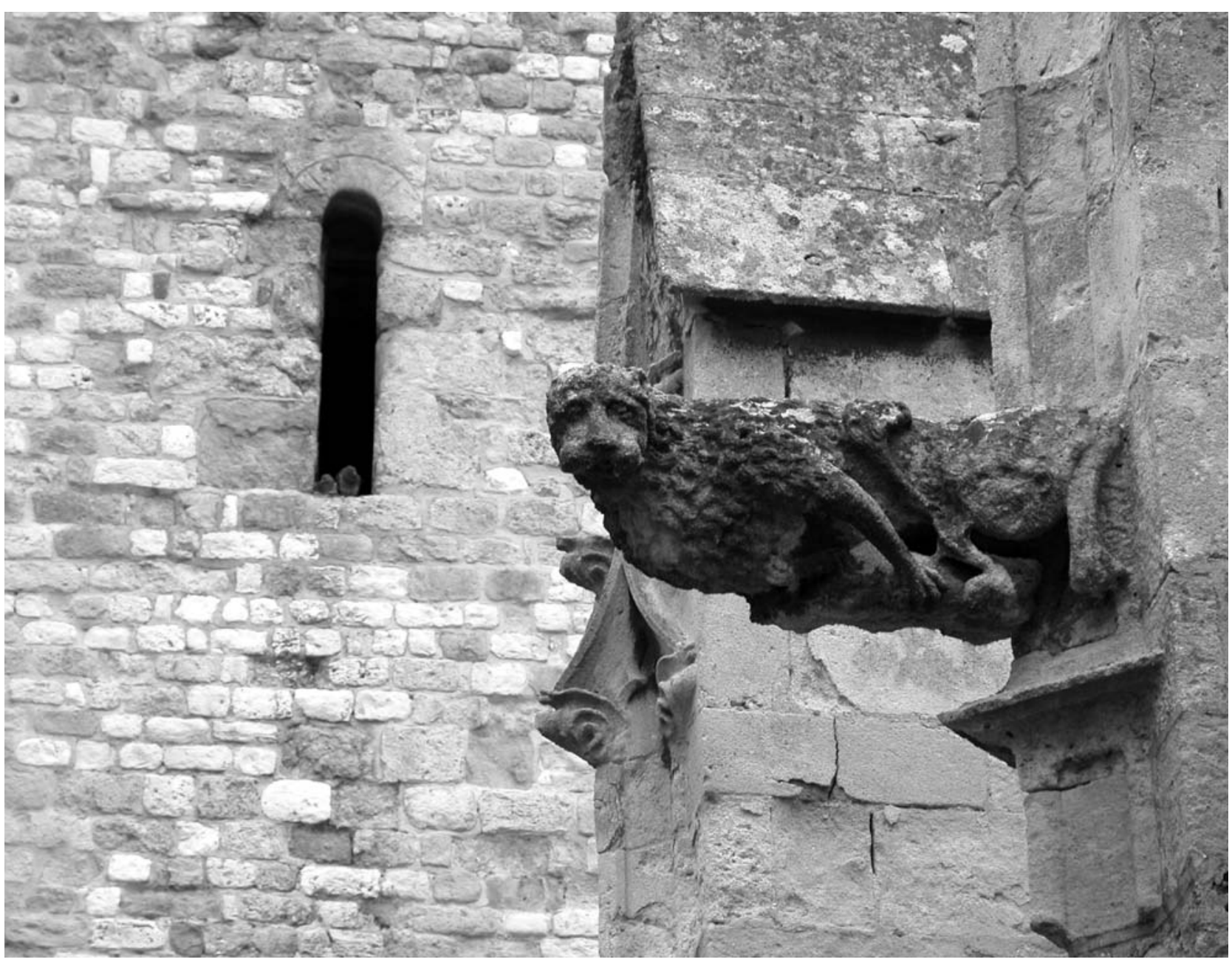

Narbonne - France 